\title{
Miossec, J.-M. (2016). Le conteneur et la nowvelle géographie des océans et des rivages de la mer. Dans le sillage de la CMA CGM. Paris, France : L'Harmattan, 724 p.
}

Martin Simard ${ }^{\mathrm{a}}$

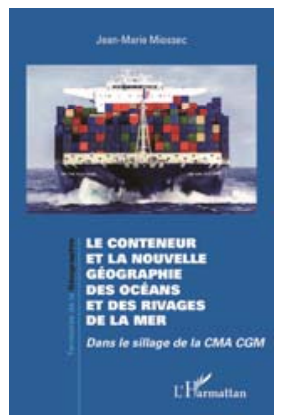

Le géographe d'expérience et ancien recteur de l'Université Paul Valéry de Montpellier, Jean-Marie Miossec, nous propose ici un ouvrage fort impressionnant. Ce livre de grande taille, qui peut intéresser tant les universitaires que les gens d'affaires, utilise l'image du conteneur pour illustrer le phénomène de la mondialisation, lequel se réalise concrètement par la maritimisation intense des échanges commerciaux. L'analyse des réseaux d'échanges maritimes et des pratiques des grands armateurs-opérateurs indiquent la montée en puissance de la Chine ainsi que le caractère périphérique de l'Amérique du Sud, de l'Afrique et de certaines régions de l'Asie. À cet égard, le trafic maritime par conteneur serait davantage structurant que le transport des minerais ou du pétrole, devenant ainsi un véritable indicateur de développement. Au passage, divers enjeux ou questions sont abordés comme l'histoire du commerce maritime, les risques et défis des compagnies engagées dans ce commerce, la résurgence du piratage ou les effets territoriaux du commerce maritime en matière d'aménagement urbain ou de développement régional.

Le livre de Miossec est organisé en dix chapitres. Ceux-ci débutent souvent par des mises en contexte historique et culminent fréquemment par des descriptions fines des routes ou ports engagés dans le commerce maritime, ce qui démontre la grande érudition de l'auteur. Le premier chapitre s'apparente à une longue introduction qui explique l'évolution du commerce maritime. On y présente trois concepts qui reviennent tout au long de l'ouvrage, soit le bub (port de grande envergure), les rangées (façades portuaires continentales) et les roues de la fortune (réseaux portuaires régionaux structurés autour d'un pôle principal). Le deuxième chapitre décrit l'histoire du conteneur et fait un portrait des grands armateursopérateurs, de leur modus operandi et des défis auxquels ils sont confrontés. Le troisième chapitre traite des risques intrinsèques au commerce maritime, du rôle stratégique des grands canaux (Suez et Panama) de même que du droit de la mer. Les quatrième et cinquième chapitres tracent un portrait de la compagnie française CMA-CGM, étude de cas qui sert d'exemple pour comprendre le monde du commerce maritime et ses transformations dans le temps. Sixièmement, l'auteur présente la zone économique exclusive maritime de la France (ZEE) dont l'ampleur et la diversité sont reliées aux territoires d'outre-mer de l'hexagone, relent de la période de colonisation. En septième lieu, Miossec discute en profondeur du corridor maritime chinois, la «rangée » la plus dynamique à l'échelle mondiale, avant de souligner l'importance relative du corridor portuaire de l'Europe du Nord. Au chapitre huit, les principes du bub et de la roue de fortune sont mis en exergue à l'aide de nombreux exemples alors que la neuvième section s'attarde aux corridors portuaires périphériques et à leurs perspectives de développement. Finalement, le chapitre dix se concentre sur les relations complexes entre les villes et leurs espaces portuaires et, plus largement, à la dialectique terre-mer qui varie selon les géographies fluviales et ferroviaires.

a Professeur, Université du Québec à Chicoutimi 
Le livre de Jean-Marie Miossec est un projet ambitieux, voire l'œuvre maittresse d'une carrière universitaire longue et fructueuse, des deux côtés de la Méditerranée et de l'Atlantique. Le document est d'une grande richesse relativement au contenu, à la fois par ses informations factuelles inédites que par la généralisation théorique sous la forme de différentes typologies. Néanmoins, quelques faiblesses subsistent comme dans toute réalisation de cette dimension. Tout d'abord, la taille du livre est plutôt hors norme. Cela devient un problème dans la mesure où certains éléments semblent non essentiels. Ainsi, à notre avis, le portrait de la firme CMA-CGM dépasse l'exemplarité pour inclure des aspects anecdotiques et certains élans patriotiques. On peut penser qu'une monographie d'entreprise aurait pu être effectuée en parallèle afin d'alléger le livre aux horizons plus vastes. Par ailleurs, Miossec aborde peu ou aucunement les effets environnementaux des aménagements portuaires ou du transport maritime de même que la question des conditions de travail déplorables de la main d'œuvre opérant les grands navires. Ces thématiques me paraissent pourtant des plus importantes. Sur le plan de la forme, on peut dire que les cartes et figures sont de qualité inégale, passant de la carte sommaire en noir et blanc aux tableaux sophistiqués de multiples couleurs. Finalement, un effort supplémentaire aurait pu être fait pour proposer des termes francophones comme équivalent des expressions anglaises, lesquelles sont largement employées dans le texte.

Dans l'ensemble, la «brique » de Jean-Marie Miossec est un livre-clef pour les observateurs ou les acteurs de la scène maritime. Il comporte une mine d'information à découvrir. En effet, un accès privilégié aux données d'une grande entreprise a permis de révéler les stratégies et la logistique de l'industrie du commerce maritime par conteneur. De surcroît, le lecteur persévérant y trouvera des connaissances fondamentales sur le commerce international, la géopolitique et le droit de la mer. 